\title{
Real-Life Clinical Data of Lenvatinib versus Sorafenib for Unresectable Hepatocellular Carcinoma in Italy
}

\author{
Valentina Burgio, (D)' Massimo \\ lavarone, ${ }^{2}$ Giovanni Giuseppe Di \\ Costanzo, ${ }^{3}$ Fabio Marra, ${ }^{4}$ Sara \\ Lonardi, (iD ${ }^{5,6}$ Emiliano Tamburini, (iD) ${ }^{7}$ \\ Fabio Piscaglia, (D) ${ }^{8}$ Gianluca Masi, ${ }^{9,10}$ \\ Ciro Celsa, "' Francesco Giuseppe \\ Foschi, ${ }^{12}$ Marianna Silletta, ${ }^{13}$ Daniela \\ Caterina Amoruso, ${ }^{14}$ Margherita \\ Rimini, ${ }^{15}$ Mariangela Bruccoleri, (iD) 2 \\ Raffaella Tortora, ${ }^{3}$ Claudia \\ Campani, (iD ${ }^{4}$ Caterina Soldà, ${ }^{6}$ \\ Massimo Giuseppe Viola, ${ }^{16}$ Antonella \\ Forgione, ${ }^{8}$ Fabio Conti, ${ }^{12}$ Francesca \\ Salani, ${ }^{9,10}$ Silvia Catanese,,${ }^{9} 10$ Carmelo \\ Marco Giacchetto, ${ }^{17}$ Claudia \\ Fulgenzi, ${ }^{13}$ Carmine Coppola, ${ }^{14}$ Pietro \\ Lampertico, ${ }^{18}$ Antonio Pellino, 6,19 \\ Gabriele Rancatore, (iD) 17 Giuseppe \\ Cabibbo, ${ }^{17}$ Francesca Ratti, ${ }^{20}$ Federica \\ Pedica, ${ }^{21}$ Angelo Della Corte, ${ }^{22}$ \\ Massimo Colombo, ${ }^{18}$ Francesco De \\ Cobelli, ${ }^{23}$ Luca Aldrighetti, ${ }^{20}$ Stefano \\ Cascinu, ${ }^{1,23}$ Andrea Casadei- \\ Gardini (iD) 1,23
}

'Department of Medical Oncology, Vita-Salute San Raffaele University, IRCCS San Raffaele Scientific Institute, Milan, 20132, Italy;

${ }^{2}$ Foundation IRCCS Ca' Granda Ospedale Maggiore Policlinico, Division of Gastroenterology and Hepatology, Milan, Italy; ${ }^{3}$ Department of Hepatology, Naples, 80I 3 I, Italy; ${ }^{4}$ Dipartimento di Medicina Sperimentale e Clinica, Università di Firenze, Firenze, Italy; ${ }^{5}$ Early Phase Clinical Trial Unit, Department of Oncology, Veneto Institute of Oncology IOVIRCCS, Padua, Italy; ${ }^{6}$ Medical Oncology Unit I, Department of Oncology, Veneto Institute of Oncology IOV - IRCCS, Padua, Italy; ${ }^{7}$ Department of Oncology and Palliative Care, Cardinale Hospital, Naples, Italy; ${ }^{8}$ Division of Internal Medicine, Hepatobiliary and Immunoallergic Disease, IRCCS Azienda OspedalieroUniversitaria di Bologna, Bologna, Italy; ${ }^{9}$ Department of Translational Research and New Technologies in Medicine and Surgery, University of Pisa, Pisa, Italy; ${ }^{10}$ Unit of Medical Oncology 2, Azienda Ospedaliero-Universitaria Pisana, Pisa, Italy; " Department of Surgical, Oncological and Oral Sciences (Di.Chir.On.S.), University of Palermo, Palermo, 90127, Italy;
Background: Lenvatinib has been approved in Italy since October 2019 as a first-line therapy for advanced hepatocellular carcinoma (HCC) and to date data on effectiveness and safety of lenvatinib are not available in our region. To fill this gap, we performed a multicentric analysis of the real-world treatment outcomes with the propensity score matching in a cohort of Italian patients with unresectable HCC who were treated with either sorafenib or lenvatinib.

Aims and Methods: To evaluate the effectiveness of sorafenib and lenvatinib as primary treatment of advanced HCC in clinical practice we performed a multicentric analysis of the treatment outcomes of 288 such patients recruited in 11 centers in Italy. A propensity score was used to mitigate confounding due to referral biases in the assessment of mortality and progression-free survival.

Results: Over a follow-up period of 11 months the Cox regression model showed $48 \%$ reduction of death risk for patients treated with lenvatinib (95\% CI: $0.34-0.81 ; p=0.0034)$, compared with those treated with sorafenib. The median PFS was 9.0 and 4.9 months for lenvatinib and sorafenib arm, respectively. Patients treated with lenvatinib showed a higher percentage of response rate $(29.4 \%$ vs $2.8 \%$; p < 0.00001$)$ compared with patients treated with sorafenib. Sorafenib was shown to be correlated with more HFSR, diarrhea and fatigue, while lenvatinib with more hypertension and fatigue.

Conclusion: Our study highlighted for the first time the efficacy and safety of lenvatinib in an Italian cohort of patients.

Keywords: hepatocarcinoma, sorafenib, lenvatinib

\section{Introduction}

Hepatocellular carcinoma (HCC) is the fourth leading cause of cancer death worldwide. $^{1}$

In the last few years, the first line treatment of advanced HCC has expanded from the pioneer multikinase inhibitor sorafenib ${ }^{2}$ to embrace lenvatinib, ${ }^{3-5}$ another multikinase inhibitor, and, more recently, a combination between the monoclonal antibody bevacizumab, that inhibits vascular endothelial growth factor (VEGF), and the immune check point inhibitor atezolizumab. ${ }^{6}$ While the registration trial REFLECT showed lenvatinib to be non-inferior to sorafenib, ${ }^{3}$ with some nuances in tolerability, in the registration trial the combination atezolizumab-bevacizumab was shown to significantly outperform the standard of care of sorafenib in terms of overall survival and radiological response. While the hierarchy of anticancer activity between this latter regimen and the multikinase inhibitors sorafenib and 
${ }^{12}$ Internal Medicine, Infermi Hospital, Faenza (AUSL ROMAGNA), Ravenna, Italy;

${ }^{13}$ Department of Oncology, Campus Bio Medico, Roma, Italy; ${ }^{14}$ Hepatology Unit, Internal Medicine, Area Stabiese Hospital, Naples, Italy;

${ }^{15}$ Department of Oncology and Hematology, Division of Oncology, University of Modena and Reggio Emilia, Modena, 4I2I, Italy; ${ }^{16}$ Department of General Surgery and Emergency Surgery, Cardinale Hospital, Tricase, Italy; ${ }^{17}$ Section of Gastroenterology \& Hepatology, Department of Health Promotion, Mother and Child Care, Internal Medicine and Medical Specialties, PROMISE, University of Palermo, Palermo, 90127, Italy; ${ }^{18}$ Liver Center, IRCCS San Raffaele Scientific Institute, Milan, 20132, Italy; ${ }^{9}$ Department of Surgery, Oncology and Gastroenterology, University of Padua, Padua, Italy; ${ }^{20}$ Hepatobiliary Surgery Division, Liver Center, Vita-Salute San Raffaele University, IRCCS San Raffaele Scientific Institute, Milan, 20132, Italy; ${ }^{21}$ Department of Experimental Oncology, Pathology Unit, IRCCS San Raffaele Scientific Institute, Milan, 20132, Italy; ${ }^{22}$ Department of Radiology, Vita-Salute San Raffaele University, IRCCS San Raffaele Scientific Institute, Milan, 20132, Italy; ${ }^{23}$ School of Medicine, Vita-Salute San Raffaele University, Milan, 20132, Italy
Correspondence: Andrea CasadeiGardini

Department of Medical Oncology, IRCCS San Raffaele Hospital, Via Olgettina n. 60, Milan, Italy Email casadeigardini@gmail.com lenvatinib is crystal clear to many, the worldwide market penetration of the combination atezolizumab-bevacizumab is rather limited due to a number of reasons, thus making a majority of patients still receive either sorafenib or lenvatinib as a first line therapeutic option for advanced HCC. Along this line, however, there remain uncertainties on which multikinase inhibitor to choose as first line treatment of advanced HCC, mainly in consideration of the different profile of tolerability and the many nuances in disease etiology, stage and ethnicity among the populations enrolled in registration trials, that have prevented a release of a clearcut recommendation. Hence, a comparative assessment of the two multikinase inhibitors in real life patients with advanced HCC might provide useful insights on the pattern of response and tolerability of these anticancer drugs that could have been obscured in the trial practice, at the same time helping to refine the therapeutic algorithm of advanced HCC.

After the result of the phase 3 trial, several real-life studies have come out with the aim of integrating the study from the phase 3 trial. ${ }^{7-11}$ These studies, mainly from eastern populations of patients, highlighted a possible superiority of lenvatinib in patients in an early stage compared to sorafenib. However, real-world data from western populations of patients is lacking. Lenvatinib has been approved in Italy since October 2019 and to date we do not have data about the activity and safety of lenvatinib in our region.

With the aim to fill this gap, we performed a multicentric analysis with the propensity score matching to compare the real-world treatment outcomes between sorafenib and lenvatinib in a cohort of Italian patients with unresectable HCC.

\section{Methods and Materials}

The study population is derived from prospectively collected data of patients treated with sorafenib or lenvatinib as a first-line for advanced-stage HCC (BCLC-C) or intermediate HCC (BCLC-B) deemed not eligible for first- or for re-treatment with surgical or loco-regional therapies.

The overall cohort included 466 consecutive patients from Italy between March 2016 and March 2021, 322 patients treated with sorafenib, 144 patients treated with lenvatinib.

33 patients treated with Sorafenib were excluded for vp4 thrombosis and/or 50\% or higher liver occupation because lenvatinib cannot be prescribed in these patients. Among the 433 selected patients, 289 were treated with sorafenib, while 144 received lenvatinib, according to the policy of each center (Figure 1).

Eligible patients had HCC diagnoses confirmed histologically or confirmed clinically in accordance with international guidelines and none of them received previous systemic therapy. Common inclusion criteria for the use of sorafenib or lenvatinib were applied.

The present study was approved by ethics committees at each centre (San Raffaele ethical committee number of protocol 113/INT/2021), complied with the provisions of the Good Clinical Practice guidelines and the Declaration of Helsinki and local laws and fulfilled the Regulation (EU) 2016/679 of the European Parliament and of the Council of 27 April 2016 on the protection of natural persons with regard to the processing of personal data. All patients provided written informed consent. 


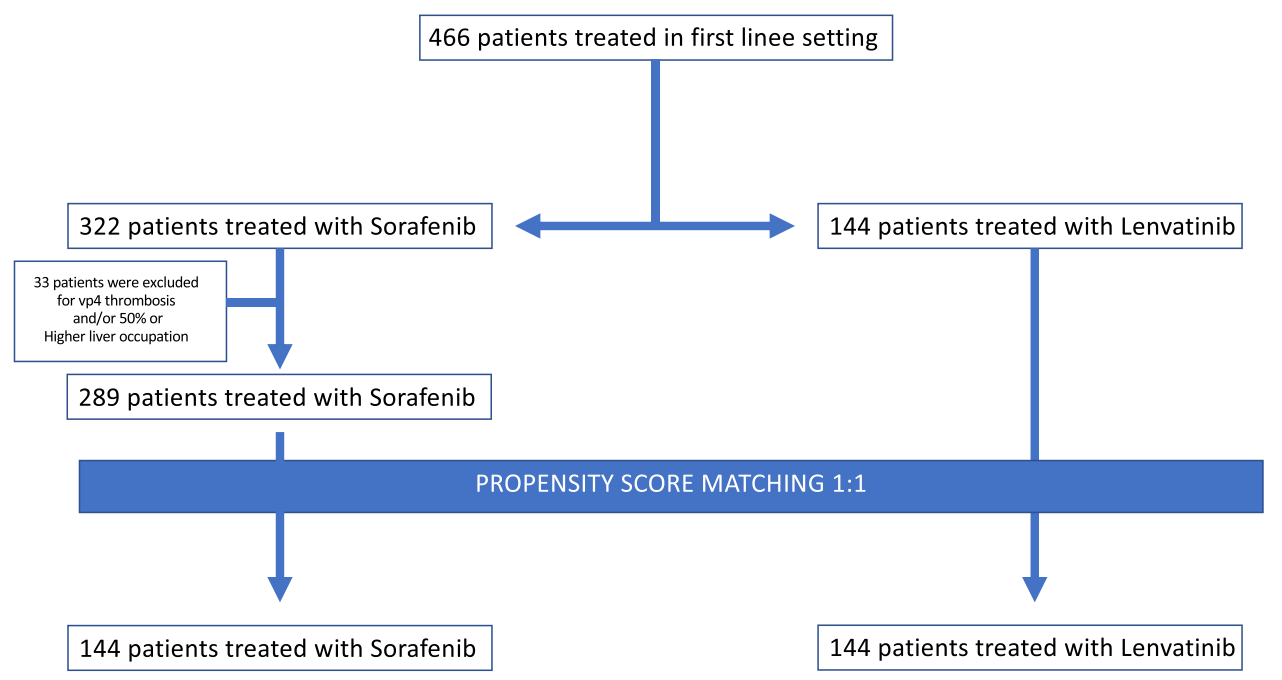

Figure I CONSORT diagram of the study.

Follow-up ended in March 2021.

Patients were treated with sorafenib or lenvatinib. The choice between the two therapies was left to physician incharge's discretion. Lenvatinib was administered as described in the REFLECT trial, thus patients received $12 \mathrm{mg}$ if baseline bodyweight was $\geq 60 \mathrm{~kg}$ or $8 \mathrm{mg}$ if baseline bodyweight was $<60 \mathrm{~kg}$, given once daily orally. ${ }^{3}$ Sorafenib was administered as in common clinical practice, and all patients in the sorafenib group received a starting dose of $400 \mathrm{mg}$ orally twice daily. ${ }^{2}$

Treatment interruptions and dose reductions were allowed to manage adverse events (AEs). Hand-foot skin reaction (HFSR), diarrhoea, hypertension, fatigue, decreased appetite, proteinuria and hypothyroidism were the main AEs of interest and were graded using the National Cancer Institute Common Terminology Criteria for Adverse Events (NCI-CTCAE) version 4.03.

\section{Statistical Analysis}

Frequency tables were performed for categorical variables. Continuous variables were presented using median and range. Overall survival (OS) was defined as the time from start date of sorafenib or lenvatinib to date of death. Progression-free survival (PFS) was defined as the time from start date of sorafenib or lenvatinib to date of progression or death or last follow-up whichever occurred first. OS and PFS were reported as median values expressed in months, with $95 \%$ confidence interval (CI). Survival curves were estimated using the product-limit method of Kaplan-Meier. The role of stratification factor was analyzed with log rank tests. Propensity score (PS) is the conditional probability of being treated given a set of observed potential confounders. In this way all the information from a group of potential confounders is summarized into a single balancing score variable, the so-called PS. PS assures that the distribution of measured baseline covariates is maintained unchanged in both arms. Standardized difference was used as a balance measure to compare the difference in means in units of the pooled standard deviation.

The analysis was performed using known cut-offs in literature for advanced-stage HCC (BCLC-C) or intermediate HCC (BCLC-B) deemed not eligible for first- or for re-treatment with surgical or loco-regional therapies (eg AFP $><400$ and NLR $><3$ ).

A MedCalc package (MedCalc ${ }^{\circledR}$ version 16.8.4) was used for statistical analysis.

\section{Results}

The main characteristics of the study population are reported in Table 1. Distribution of child-pugh $(\mathrm{p}=0.03)$ and ECOG performance status $(p=0.000025)$ was more favorable in lenvatinib than in sorafenib patients. Conversely, normal bilirubin value $(\mathrm{p}=0.006)$ was more favorable in sorafenib than in lenvatinib patients (Table 1).

To minimize the confounding effect of the uneven distribution of baseline characteristics, a propensity score matching was performed. Lenvatinib and sorafenib patients were matched with propensity matching analysis.

Sorafenib and lenvatinib patients were matched for ECOG performance status. The matching allowed us to 
Table I Patient Characteristics Before and After Propensity Score Matching

\begin{tabular}{|c|c|c|c|c|c|c|}
\hline & \multicolumn{3}{|c|}{ Before Propensity Score Matching } & \multicolumn{3}{|c|}{ After Propensity Score Matching } \\
\hline & $\begin{array}{c}\text { Sorafenib } \\
\% \\
\text { N = } \mathbf{2 8 9} \text { Patients }\end{array}$ & $\begin{array}{c}\text { Lenvatinib } \\
\% \\
\text { N = I44 Patients }\end{array}$ & $P$ value & $\begin{array}{c}\text { Sorafenib } \\
\% \\
\text { N = I44 Patients }\end{array}$ & $\begin{array}{c}\text { Lenvatinib } \\
\% \\
\text { N = I44 Patients }\end{array}$ & $P$ value \\
\hline Gender & & & & & & \\
\hline Male & 85.5 & 77.1 & & 82.6 & 77.1 & 0.24 \\
\hline Female & 14.5 & 22.9 & 0.48 & 17.4 & 22.9 & \\
\hline Age & & & & & & \\
\hline$<70$ & 54.0 & 52.8 & & 52.7 & 52.8 & 1.00 \\
\hline$>70$ & 46.0 & 47.2 & 0.83 & 68 & 47.2 & \\
\hline Etiology & & & & & & \\
\hline $\mathrm{HCV}$ & 48.8 & 46.5 & & 48.6 & 46.5 & 0.34 \\
\hline HBV & 20.8 & 15.2 & & 21.5 & 15.2 & \\
\hline NASH & 11.1 & 13.9 & & 12.5 & 13.9 & \\
\hline Others & 19.3 & 24.4 & 0.33 & 17.4 & 24.4 & \\
\hline TACE before & & & & & & \\
\hline Yes & 44.3 & 41.0 & & 41.0 & 41.0 & 1.00 \\
\hline No & 55.7 & 59.0 & 0.53 & 59.0 & 59.0 & \\
\hline Child-Pugh & & & & & & \\
\hline A & 88.6 & 94.9 & & 93.0 & 94.9 & 0.61 \\
\hline B & II.4 & 5.1 & 0.03 & 7.0 & 5.1 & \\
\hline $\mathrm{BCLC}$ & & & & & & \\
\hline C & 81.3 & 75.0 & & 75.0 & 75.0 & 1.00 \\
\hline B & 18.7 & 25.0 & 0.12 & 25.0 & 25.0 & \\
\hline ECOG & & & & & & \\
\hline 0 & 58.5 & 78.9 & & 78.9 & 78.9 & 1.00 \\
\hline$>0$ & 41.5 & 21.1 & 0.000025 & 21.1 & 21.1 & \\
\hline AFP & & & & & & \\
\hline$<400$ & 69.2 & 64.6 & & 69.3 & 64.6 & 0.43 \\
\hline$>400$ & 30.8 & 35.4 & 0.07 & 30.7 & 35.4 & \\
\hline NLR & & & & & & \\
\hline$<3$ & 60.4 & 65.1 & & 65.4 & 65.1 & 1.00 \\
\hline$>3$ & 39.6 & 34.9 & 0.38 & 34.6 & 34.9 & \\
\hline Bilirubin & & & & & & \\
\hline$<N V$ & 80.1 & 64.0 & & 66.9 & 64.0 & 0.70 \\
\hline$>\mathrm{NV}$ & 19.9 & 36.0 & 0.006 & 33.1 & 36.0 & \\
\hline Albumin & & & & & & \\
\hline$<35$ & 24.2 & 13.5 & & 21.3 & 13.5 & 0.13 \\
\hline$>35$ & 75.8 & 86.5 & 0.53 & 78.7 & 86.5 & \\
\hline
\end{tabular}

select 144 pairs of patients (1:1 case-control matching) homogeneous for all baseline characteristics (Table 1).

After matching, median OS was not reached for patients receiving lenvatinib and was 12.0 months $(95 \%$ CI: 9.7-29.1) for patients treated with sorafenib (Figure 2A). The result from univariate Cox regression model showed $48 \%$ reduction of death risk for patients treated with lenvatinib (95\% CI: 0.34-0.81; $\mathrm{p}=0.0034$ ), compared with patients on sorafenib. Furthermore, at univariate analysis, alpha-fetoprotein, BCLC stage, child pugh and portal vein thrombosis were associated with overall survival (Table 2). 

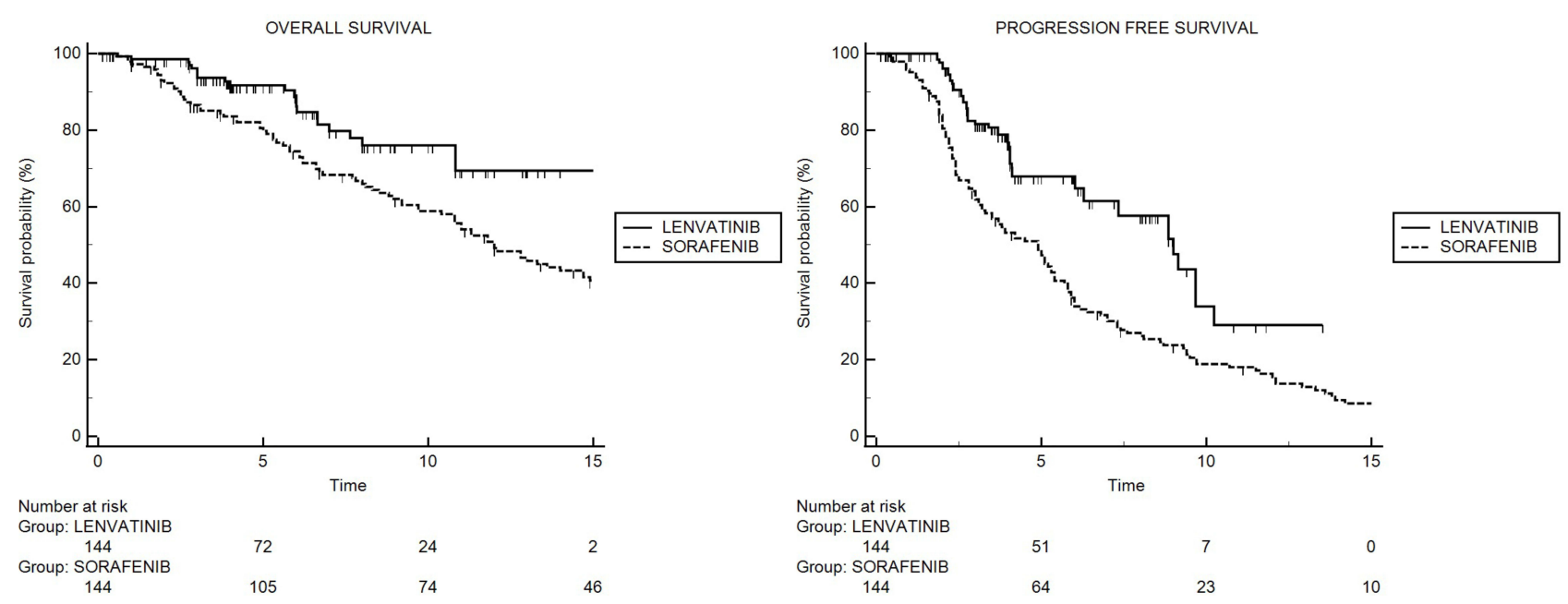

Figure 2 Kaplan-Meier curves for OS in sorafenib and lenvatinib cohorts (A), and Kaplan-Meier curves for PFS in sorafenib and lenvatinib cohorts (B).

Following adjustment for clinical covariates positive in univariate analysis, multivariate analysis confirmed lenvatinib versus sorafenib (HR 0.54; 95\% CI: $0.27-0.73$; $\mathrm{p}=$ 0.0115), as independent prognostic factor for OS (Table 3 ).

After matching, median PFS was 9.0 months $(95 \% \mathrm{CI}$ : 7.3-10.2) for patients receiving lenvatinib and 4.9 months (95\% CI: 3.3-43.4) for patients treated with sorafenib (Figure 2B). The result from univariate Cox regression model showed $51 \%$ reduction of progression risk for patients on lenvatinib (95\% CI: 0.27-0.66; p < 0.0001), compared with patients on sorafenib.

After matching, patients treated with lenvatinib showed a higher percentage of response rate (29.4 vs 2.8; $\mathrm{p}<0.00001)$ compared to patients treated with sorafenib; no differences were found in terms of disease control rate (lenvatinib 76.7 vs sorafenib 67.8; $\mathrm{p}=0.13$ ) (Figure 3).

Table 3 reports the adverse events (AEs) observed. Overall, $97.3 \%$ and $97.9 \%$ experienced at least one (any grade) AE in lenvatinib and sorafenib arm, respectively. The main drug-related AEs in the lenvatinib arm were fatigue $(41.0 \%)$, hypertension $\quad(35.4 \%)$ and decreased appetite (32.6\%). Conversely, the main drugrelated AEs in the sorafenib arm were HFSR (38.2\%), diarrhea (38.2) and fatigue (29.2). Data highlighted that HFSR $(p=0.000001)$ and diarrhea grade $>2(p=0.02)$ were significantly more frequent in patients treated with sorafenib, while hypertension grade $>2(p=0.000001)$ and fatigue $(p=0.04)$ were significantly more frequent in patients treated with lenvatinib. Treatment dose reduction was performed in 41 patients (28.5\%) treated with lenvatinib and in 48 patients treated with sorafenib (28.5\%).
Forest plot highlighted that lenvatinib had a better OS with respect to sorafenib in patients aged $>70$ years, BCLC $\mathrm{B}$ and $\mathrm{C}$ stage, nonalcoholic steatohepatitis (NASH) correlate, presence of portal vein thrombosis (PVT), alphafetoprotein (AFP) $<400$, neutrophil-lymphocyte ratio (NLR) $<3$ and $>3$ and ECOG 0 (Figure 4A). Forest plot also highlighted that lenvatinib had better PFS in all subcategories except for patients with $\mathrm{HCV}$ and $\mathrm{HBV}$ positive cirrhosis, AFP $>400$ and ECOG $>0$ (Figure 4B).

\section{Discussion}

Firstly, this analysis based on real-world data from an Italian cohort of patients demonstrated a significant advantage in terms of overall survival of lenvatinib compared to sorafenib. To our knowledge this is the first study conducted in a real-life setting of Italian patients. Secondly, median progression free survival was significantly better in patients treated with lenvatinib compared with those treated with sorafenib (9.0 vs 4.0 months respectively), confirming as reported in the REFLECT trial. ${ }^{3}$ Also, with regard to response rate, the results of our analysis are consistent with those of the REFLECT trial, finding a higher percentage of response rates in patients treated with lenvatinib compared to patients treated with sorafenib. ${ }^{3}$ This advantage has not only a robust statistical value but also great clinical value. Another aspect of discussion is the safety profile, in our study the incidence of AEs of any grade was similar in the two arms of treatments, with a difference in terms of type of toxicity profile: in the sorafenib group there was a higher incidence of HFSR, diarrhea and fatigue while in the lenvatinib group 
Table 2 Univariate and Multivariate Cox Analysis of Factors Associated with Mortality

\begin{tabular}{|c|c|c|c|c|}
\hline \multirow[b]{2}{*}{$\begin{array}{l}\text { Treatment } \\
\text { Sorafenib } \\
\text { Lenvatinib }\end{array}$} & \multicolumn{2}{|c|}{ Univariate Analysis } & \multicolumn{2}{|c|}{ Multivariate Analysis } \\
\hline & $\begin{array}{l}\text { I } \\
0.52(0.34-0.8 I)\end{array}$ & 0.0034 & $\begin{array}{l}\text { I } \\
0.54(0.27-0.73)\end{array}$ & 0.0115 \\
\hline $\begin{array}{l}\text { Alpha-fetoprotein } \\
<400 \\
>400\end{array}$ & $\begin{array}{l}\text { I } \\
\text { I.7I (1.|4-2.55) }\end{array}$ & 0.0083 & $\begin{array}{l}\text { I } \\
\text { I.79 (I.22-2.63) }\end{array}$ & 0.0030 \\
\hline $\begin{array}{l}\text { BCLC } \\
B \\
C\end{array}$ & $1.60(1.10-2.33)$ & 0.0127 & $1.78(1.11-2.85)$ & 0.0153 \\
\hline $\begin{array}{l}\text { Child Pugh } \\
\text { A } \\
\text { B }\end{array}$ & $\begin{array}{l}\text { I } \\
18.08(6.39-51.1)\end{array}$ & $<0.0001$ & $4.49(2.49-8.09)$ & $<0.0001$ \\
\hline $\begin{array}{l}\text { Etiology } \\
\mathrm{HCV} \\
\mathrm{HBV} \\
\text { NASH } \\
\text { Others }\end{array}$ & $\begin{array}{l}\text { I } \\
\text { I.23(0.80-I.9I) } \\
0.90(0.39-2.03) \\
\text { I.54 (0.94-2.52) }\end{array}$ & 0.21 & & \\
\hline $\begin{array}{l}\text { NLR } \\
<3 \\
>3\end{array}$ & $1.09(0.74-1.60)$ & 0.65 & & \\
\hline $\begin{array}{l}\text { Portal vein thrombosis } \\
\text { No } \\
\text { Yes }\end{array}$ & $1.99(1.35-2.90)$ & 0.0004 & & \\
\hline $\begin{array}{l}\text { ECOG } \\
0 \\
>0\end{array}$ & I. $39(0.87-2.20)$ & 0.1610 & & \\
\hline
\end{tabular}

there was a higher incidence of fatigue, hypertension and decreased appetite. No new toxicities were found.

Improvements for both survival parameters (OS and PFS) with lenvatinib compared to sorafenib were observed across patients' subgroups. Lenvatinib showed a better PFS in all subcategories except patients with $\mathrm{HCV}$ and HBV positive cirrhosis, AFP value $>400$ and ECOG $>0$.

Notably, BCLC B and C stage, NASH, age $>70$ and NLR were reported to be all characteristics in favor of treatment with lenvatinib for better OS and PFS. In REFLECT, BCLC C stage demonstrated favoring lenvatinib with respect to sorafenib for only PFS, underlying the major benefit of lenvatinib in the subgroup of patients with an advanced stage of disease. In our analysis the advantage of lenvatinib was observed also in patients with an intermediate stage of disease, in particular in patients with TACE refractoriness. ${ }^{3}$ This data is in line with the recent papers published on lenvatinib in a real-life setting. These studies highlighted a beneficial effect of lenvatinib as an early treatment in TACE-refractory disease. ${ }^{7-9}$ This data was recently confirmed by our group in a large population of eastern patients, where lenvatinib was highlighted as having a longer survival than sorafenib in patients previously treated with TACE. ${ }^{10}$ Moreover, Kudo et al have demonstrated the superiority of lenvatinib compared to TACE in patients with intermediate-stage hepatocellular carcinoma beyond up-to-seven criteria; ${ }^{11}$ data on the Italian cohort of patients reinforced this concept.

Interestingly, our study highlighted the benefit of lenvatinib over sorafenib both in terms of progression free survival and overall survival in patients with NASH. Data about the role of NASH in HCC patients treated with lenvatinib are lacking in the literature, and in particular forest plot analysis from the REFLECT trial did not 
Table 3 Adverse Events in Sorafenib and Lenvatinib Arms

\begin{tabular}{|c|c|c|c|}
\hline & $\begin{array}{c}\text { Lenvatinib Arm } \\
\%\end{array}$ & $\begin{array}{c}\text { Sorafenib Arm } \\
\%\end{array}$ & $\mathbf{P}$ \\
\hline \multicolumn{4}{|c|}{ All toxicity } \\
\hline No & 2.8 & 2.1 & 1.00 \\
\hline Yes & 97.2 & 97.9 & \\
\hline \multicolumn{4}{|c|}{ GRADE } \\
\hline $\mathrm{I}-2$ & 67.8 & 72.3 & 0.43 \\
\hline$>2$ & 29.4 & 25.6 & \\
\hline \multicolumn{4}{|c|}{ HFSR } \\
\hline No & 89.5 & 61.8 & $<0.000001$ \\
\hline Yes & 10.5 & 38.2 & \\
\hline \multicolumn{4}{|c|}{ GRADE } \\
\hline $\mathrm{I}-2$ & 5.5 & 29.9 & 0.17 \\
\hline$>2$ & 5.0 & 8.3 & \\
\hline \multicolumn{4}{|c|}{ Diarrhea } \\
\hline No & 72.9 & 69.5 & 0.60 \\
\hline Yes & 37.1 & 30.5 & \\
\hline \multicolumn{4}{|c|}{ GRADE } \\
\hline $\mathrm{I}-2$ & 37.1 & 26.4 & 0.02 \\
\hline$>2$ & 0.0 & 4.1 & \\
\hline \multicolumn{4}{|c|}{ Hypertension } \\
\hline No & 64.6 & 75.7 & 0.053 \\
\hline Yes & 35.4 & 24.3 & \\
\hline \multicolumn{4}{|c|}{ GRADE } \\
\hline $\mathrm{I}-2$ & 9.0 & 19.4 & 0.000001 \\
\hline$>2$ & 26.4 & 4.9 & \\
\hline \multicolumn{4}{|c|}{ Fatigue } \\
\hline No & 59.0 & 70.8 & 0.04 \\
\hline Yes & 41.0 & 29.2 & \\
\hline \multicolumn{4}{|c|}{ GRADE } \\
\hline $1-2$ & 25.0 & 24.3 & 0.01 \\
\hline$>2$ & 16.0 & 4.9 & \\
\hline \multicolumn{4}{|c|}{ Decrease appetite } \\
\hline No & 67.4 & NR & \\
\hline Yes & 32.6 & NR & \\
\hline \multicolumn{4}{|c|}{ GRADE } \\
\hline $\mathrm{I}-2$ & 20.1 & NR & \\
\hline$>2$ & 12.5 & NR & \\
\hline \multicolumn{4}{|c|}{ Proteinuria } \\
\hline No & 93.0 & NR & \\
\hline Yes & 7.0 & NR & \\
\hline \multicolumn{4}{|c|}{ GRADE } \\
\hline $1-2$ & 4.2 & NR & \\
\hline$>2$ & 2.8 & NR & \\
\hline
\end{tabular}

(Continued) 
Table 3 (Continued).

\begin{tabular}{|l|c|c|c|}
\hline & $\begin{array}{c}\text { Lenvatinib Arm } \\
\%\end{array}$ & $\begin{array}{c}\text { Sorafenib Arm } \\
\%\end{array}$ & P \\
\hline Hypothyroidism & & NR \\
No & 73.6 & NR & \\
Yes & 26.4 & NR & \\
GRADE & GRADE & NR & \\
I-2 & 9.7 & & \\
$>2$ & 16.7 & 54.9 & $\mathbf{0 . 0 1}$ \\
\hline Other toxicity & & 45.1 & \\
No & 68.7 & 38.9 & \\
Yes & 31.3 & 6.2 & $\mathbf{0 . 0 0 1}$ \\
GRADE & 17.4 & & \\
I-2 & 13.9 & & \\
$>2$ & & & \\
\hline
\end{tabular}

Note: In bold font positive results.

include this information. HCC during NASH etiology grows up very fast and the observation of a clinical benefit of lenvatinib over sorafenib in this subgroup of patients is crucial for our clinical practice. This was an important finding, because recently Pfister et al highlighted that nonviral HCC, and particularly NASH-HCC, might be less responsive to immunotherapy. ${ }^{12}$

Clearly, we should do a specific study on this item to confirm this data. In patients with $\mathrm{HCV}$ infection data highlights the same efficacy of lenvatinib over sorafenib as already demonstrated in a previous metanalysis about lenvatinib and sorafenib efficacy in patients with HBV and HCV infection. ${ }^{13}$

This study highlighted the effectiveness of lenvatinib also in an older age group, probably a better toxicity profile of lenvatinib over sorafenib can explain this aspect. A previous study highlighted that lenvatinib can be used safely and efficaciously regardless of age. ${ }^{14}$

Finally, a high baseline value of NLR is widely recognized as a parameter of poor prognosis in $\mathrm{HCC}$ and its role

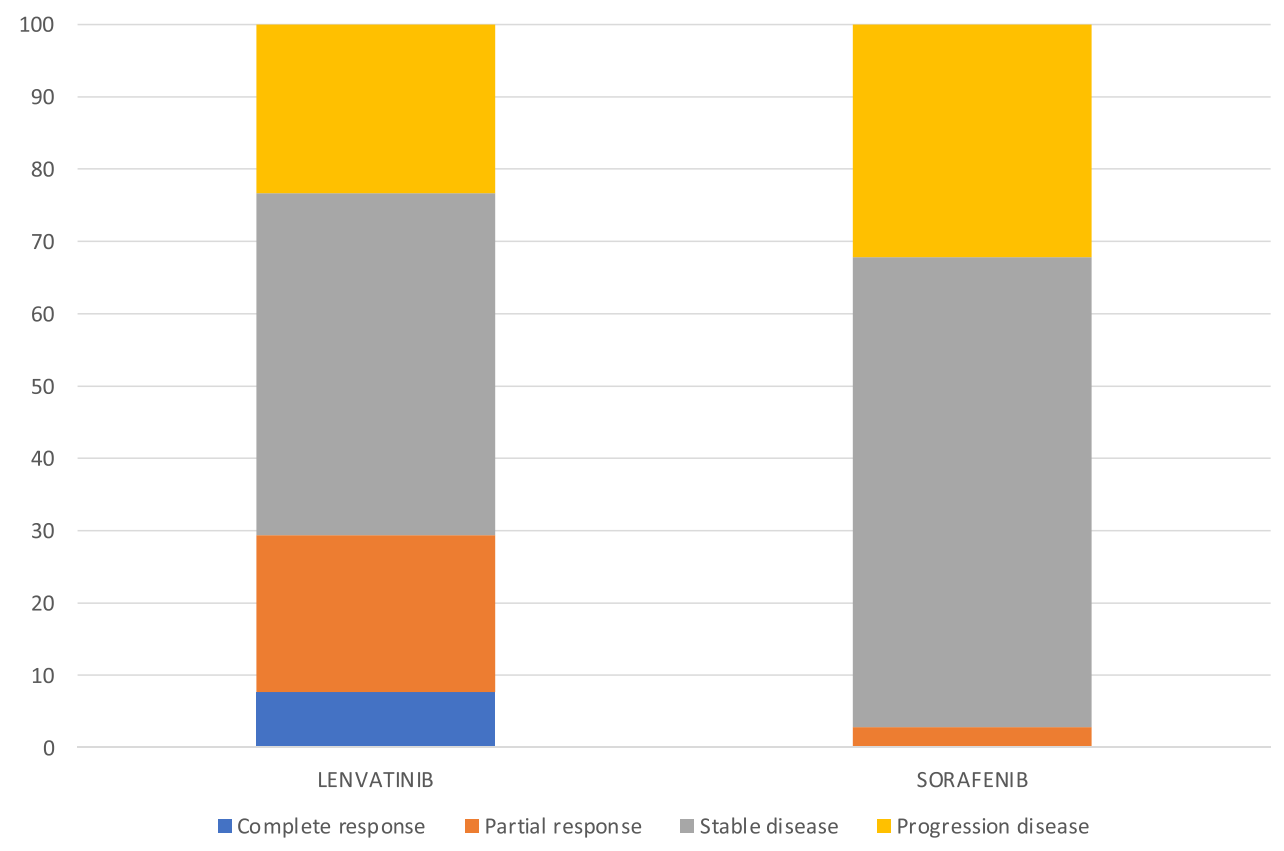

Figure 3 Different response rates between sorafenib and lenvatinib. 
OVERALL SURVIVAL

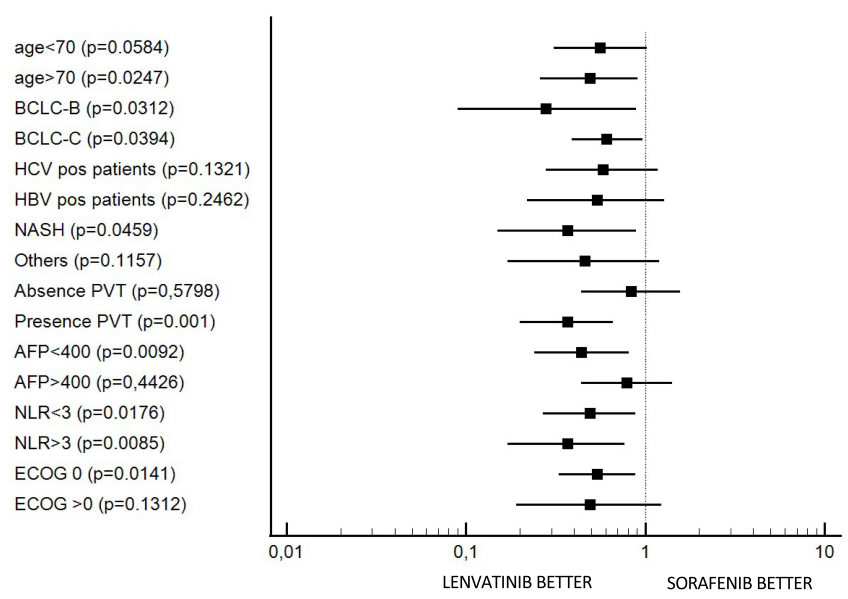

PROGRESSION FREE SURVIVAL

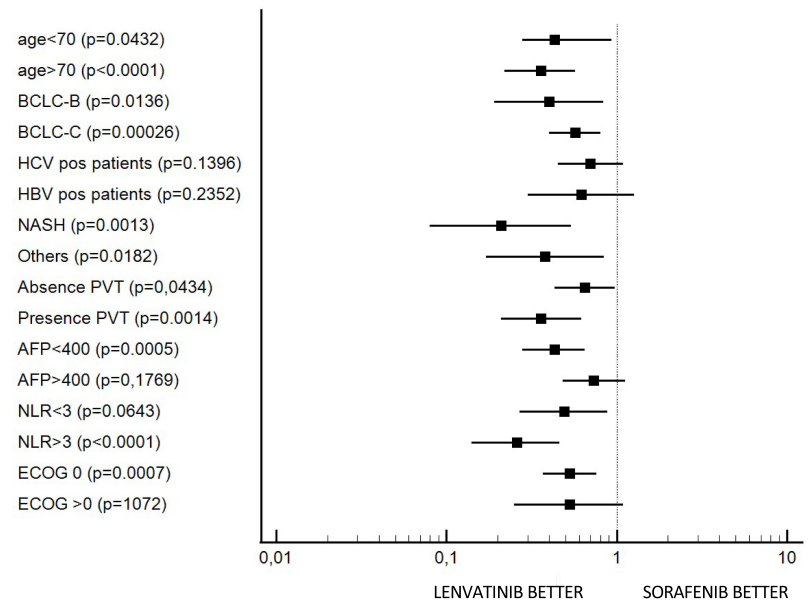

Figure 4 Forest plot for overall survival (A) and progression free survival (B).

for worsening OS has been demonstrated. ${ }^{15,16}$ In the pooled analysis of SHARP and Asia-Pacific trials, Bruix et al found a negative correlation between high NLR and outcome and in a previous multicentric retrospective study on patients with advanced HCC treated with sorafenib, to evaluate the potential role of baseline characteristics in predicting a longer survival there was found a strong correlation between a low baseline NLR and better OS. ${ }^{17-19}$ In the present study the advantage of lenvatinib over sorafenib was seen regardless of NLR, so also in a subgroup of patients who are potentially candidates for a worse prognosis because of high NLR ( $>3)$.

Finally, other propensity score-matching analysis was recently published with the same aim of evaluating sorafenib versus lenvatinib in terms both of overall survival and progression free survival. One of these was recently published, ${ }^{20}$ results of this study highlighted an increase in progression free survival of patients treated with lenvatinib and the same overall survival between the two drugs. ${ }^{20}$ This different result between our study and the study of Nakano et al could probably be due to a different population under consideration (Western versus Eastern).

Our study has several limitations. The principal ones rely on its short follow-up of patients treated with lenvatinib. Clearly, this point limits our conclusion but there was an urgent need for data on lenvatinib in an Italian cohort of patients. Another aspect was the retrospective nature of the study and on the lack of a standardized follow-up protocol in regards to clinical monitoring of $\mathrm{HCC}$, which depended on each institution's clinical practice. Furthermore, not all data about the initial stage (eg, tumor diameter or number of nodules) or follow up was collect properly (eg increased dosage after toxicity experience). Nevertheless, the present work captures real-world observational data which could help to clarify the efficacy and tolerability of lenvatinib compared to sorafenib in an advanced HCC setting. Moreover, the use of propensity score matching reduces the selection bias inherent in the nature of a retrospective trial which compares two heterogenous cohorts of patients, thus helping the understanding of the real impact of lenvatinib rather than sorafenib.

In conclusion, our study highlighted for the first time the efficacy and safety of lenvatinib in an Italian cohort of patients. Moreover, our study confirmed that in earlier patients a larger benefit might be expected from lenvatinib treatment.

\section{Highlights}

After the result of the phase 3 trial, several real-life studies have come out with the aim of integrating the study from phase 3 trials.

With the aim to fill this gap, we performed a multicentric analysis with the propensity score matching to compare the real-world treatment outcomes between sorafenib and lenvatinib in a cohort of Italian patients with unresectable HCC.

The Cox regression model showed a $48 \%$ reduction of death risk for patients treated with lenvatinib (95\% CI: 0.34-0.81; $\mathrm{p}=0.0034)$, compared with those treated with sorafenib. 


\section{Author Contributions}

Andrea Casadei-Gardini accessed all the data in the study and took responsibility for the integrity of the data and the accuracy of the data analysis. Study concept and design: All authors. Acquisition, analysis, or interpretation of data: All authors. Drafting of the manuscript: All authors. Critical revision of the manuscript for important intellectual content: All authors. Statistical analysis: Andrea Casadei-Gardini. All authors made a significant contribution to the work reported, whether that is in the conception, study design, execution, acquisition of data, analysis and interpretation, or in all these areas; took part in drafting, revising or critically reviewing the article; gave final approval of the version to be published; have agreed on the journal to which the article has been submitted; and agree to be accountable for all aspects of the work.

\section{Funding}

There is no funding to report.

\section{Disclosure}

ACG: Speaking/teaching, consultant and advisory board for Bayer, AstraZeneca, BMS, Ipsen, MSD, EISAI. MI: Speaking/teaching, consultant and advisory board for Bayer, Gilead Sciences, BMS, Janssen, Ipsen, MSD, BTGBoston Scientific, AbbVie, Guerbet, EISAI, Shionogi. FM: Consultant for and travel grants from Bayer, consultant for EISAI/Merck and Ipsen, during the conduct of the study; consultant for Menarini, Novo Nordisk, Astra Zeneca, Allergan, and Novartis, travel grants from AbbVie. MI: personal fees from IPSEN, during the conduct of the study. FP: advisory board, consultant/lectures from Bracco, Bayer, Astra Zeneca, IPSEN, Roche, Eisai, MSD, Esaote, GE, Samsung, Exact Sciences, Tiziana Life Sciences. CC: personal fees from Eisai, Ipsen. The authors report no other conflicts of interest in this work.

\section{References}

1. Bray F, Ferlay J, Soerjomataram I, Siegel RL, Torre LA, Jemal A. Global cancer statistics 2018: GLOBOCAN estimates of incidence and mortality worldwide for 36 cancers in 185 countries. CA Cancer J Clin. 2018;68(6):394-424. doi:10.3322/caac.21492

2. Llovet JM, Ricci S, Mazzaferro V, et al.; SHARP Investigators Study Group. Sorafenib in advanced hepatocellular carcinoma. $N$ Engl J Med. 2008;359(4):378-390. doi:10.1056/NEJMoa0708857

3. Kudo M, Finn RS, Qin S, et al. Lenvatinib versus sorafenib in first-line treatment of patients with unresectable hepatocellular carcinoma: a randomised phase 3 non-inferiority trial. Lancet. 2018;391 (10126):1163-1173. doi:10.1016/S0140-6736(18)30207-1
4. Tohyama O, Matsui J, Kodama K, et al. Antitumor activity of lenvatinib (e7080): an angiogenesis inhibitor that targets multiple receptor tyrosine kinases in preclinical human thyroid cancer models. J Thyroid Res. 2014;2014:638747. doi:10.1155/2014/ 638747

5. Yamamoto Y, Matsui J, Matsushima T, et al. Lenvatinib, an angiogenesis inhibitor targeting VEGFR/FGFR, shows broad antitumor activity in human tumor xenograft models associated with microvessel density and pericyte coverage. Vasc Cell. 2014;6:18. doi:10.1186/ 2045-824X-6-18

6. Finn RS, Qin S, Ikeda M, et al.; IMbrave150 Investigators. Atezolizumab plus bevacizumab in unresectable hepatocellular carcinoma. N Engl J Med. 2020;382(20):1894-1905. doi:10.1056/ NEJMoa1915745

7. Shimose S, Kawaguchi T, Tanaka M, et al. Lenvatinib prolongs the progression free survival time of patients with intermediate-stage hepatocellular carcinoma refractory to transarterial chemoembolization: a multicenter cohort study using data mining analysis. Oncol Lett. 2020;20(3):2257-2265. doi:10.3892/ol.2020.11758

8. Shimose S, Iwamoto $\mathrm{H}$, Tanaka $\mathrm{M}$, et al. Alternating lenvatinib and trans-arterial therapy prolongs overall survival in patients with inter-mediate stage hepatocellular carcinoma: a propensity score matching study. Cancers (Basel). 2021;13(1):160.

9. Lee J, Sung PS, Yang H, et al. A real-world comparative analysis of lenvatinib and sorafenib as a salvage therapy for transarterial treatments in unresectable HCC. J Clin Med. 2020;9(12):4121. doi: $10.3390 /$ jcm 9124121

10. Casadei-Gardini A, Scartozzi M, Tada T, et al. Lenvatinib versus Sorafenib in first-line treatment of unresectable hepatocellular carcinoma: an inverse probability of treatment weighting analysis. Liver Int. 2021;41:14817.

11. Kudo M, Ueshima K, Chan S, et al. Lenvatinib as an initial treatment in patients with intermediate-stage hepatocellular carcinoma beyond up-to-seven criteria and child-pugh a liver function: a proof-ofconcept study. Cancers (Basel). 2019;11(8):1084. doi:10.3390/ cancers11081084

12. Pfister D, Núñez NG, Pinyol R, et al. NASH limits anti-tumour surveillance in immunotherapy-treated HCC. Nature. 2021;592 (7854):450-456. doi:10.1038/s41586-021-03362-0

13. Casadei Gardini A, Puzzoni M, Montagnani F, et al. Profile of lenvatinib in the treatment of hepatocellular carcinoma: design, development, potential place in therapy and network meta-analysis of hepatitis B and hepatitis C in all phase III trials. Onco Targets Ther. 2019;12:2981-2988. doi:10.2147/OTT.S192572

14. Tada T, Kumada T, Hiraoka A, et al. Safety and efficacy of lenvatinib in elderly patients with unresectable hepatocellular carcinoma: a multicenter analysis with propensity score matching. Hepatol Res. 2020;50(1):75-83. doi:10.1111/hepr.13427

15. Xiao WK, Chen D, Li SQ, Fu SJ, Peng BG, Liang LJ. Prognostic significance of neutrophil-lymphocyte ratio in hepatocellular carcinoma: a meta-analysis. BMC Cancer. 2014;14:117. doi:10.1186/ 1471-2407-14-117

16. Xue TC, Zhang L, Xie XY, et al. Prognostic significance of the neutrophil-to-lymphocyte ratio in primary liver cancer: a meta-analysis. PLoS One. 2014;9(5):e96072. doi:10.1371/journal. pone.0096072

17. Bruix J, Cheng AL, Meinhardt G, Nakajima K, De Sanctis Y, Llovet J. Prognostic factors and predictors of sorafenib benefit in patients with hepatocellular carcinoma: analysis of two phase III studies. J Hepatol. 2017;67(5):999-1008. doi:10.1016/j.jhep.2017.06.026

18. Casadei-Gardini A, Dadduzio V, Rovesti G, et al. Utility of neutrophil-to-lymphocyte ratio to identify long-term survivors among HCC patients treated with sorafenib. Medicine (Baltimore). 2020;99(22):e19958. doi:10.1097/MD.0000000000 019958 
19. Casadei Gardini A, Scarpi E, Faloppi L, et al. Immune inflammation indicators and implication for immune modulation strategies in advanced hepatocellular carcinoma patients receiving sorafenib. Oncotarget. 2016;7(41):67142-67149. doi:10.18632/ oncotarget. 11565
20. Nakano M, Kuromatsu R, Niizeki T, et al.; Kurume Liver Cancer Study Group of Japan. Primary treatment with molecular-targeted agents for hepatocellular carcinoma: a propensity score-matching analysis. Hepatol Commun. 2020;4(8):1218-1228. doi:10.1002/ hep4.1535

\section{Publish your work in this journal}

Cancer Management and Research is an international, peer-reviewed open access journal focusing on cancer research and the optimal use of preventative and integrated treatment interventions to achieve improved outcomes, enhanced survival and quality of life for the cancer patient.
The manuscript management system is completely online and includes a very quick and fair peer-review system, which is all easy to use. Visit http://www.dovepress.com/testimonials.php to read real quotes from published authors. 